\title{
ASPECTOS IMPORTANTES DE LOS PROYECTOS ICT4D, HACIA UN MODELO PARA LA EVALUACIÓN DE CALIDAD
}

\author{
Peter Mozelius, ${ }^{9}$ Henrik Hansson, ${ }^{10}$ \\ Evelyn Kigozi Kahiigi, ${ }^{11}$ Love Ekenberg ${ }^{12}$
}

RESUMEN

La Tecnología de Comunicación e Información para el Desarrollo [Information and Communications Technology (ICT4D)] es un enfoque adoptado para mejorar las condiciones socioeconómicas en países pobres. Esta incluye e-learning, software abiertos y cerrados, e-democracia, medios digitales, e-gobierno, capacidad de construcción y desarrollo de infraestructura. Billones de dólares son invertidos en dichos programas, financiados por organizaciones internacionales de beneficencia, agencias financiadas por el Estado, y compañías multinacionales privadas. Algunos problemas bien conocidos son la falta de infraestructura, el crimen, y la falta de adaptación a los contextos socioculturales. Es un campo multidisciplinario, con múltiples acreedores, y un campo multimedia complejo, con un marco teórico emergente.

Este ensayo identifica y examina aspectos de calidad importantes de los proyectos ICT4D y provee ejemplos empíricos para ilustrar cada aspecto. La pregunta general de este estudio es: ¿Qué hace que algunos proyectos ICT4D sean más exitosos que otros?

Basados en la experiencia personal del autor, con proyectos en Sri Lanka, Uganda y Vietnam, así como en análisis de otros proyectos, los siguientes aspectos de calidad son considerados como cruciales para la implementación exitosa de la ICT (Tecnología de Comunicación e Información) en proyectos de desarrollo: 1) Necesidades locales auténticas; 2) Propiedad local; 3) Limitaciones reales; 4) Redes de aptitudes; 5) Estrategia de comunicación; 6) Horizonte de planeación; 7) Documentación/resultados medibles; 8) Recursos y sustentabilidad; 9) Diversión/Motivación.

La importancia de los aspectos mencionados es discutida y explicada en este artículo.

Las áreas temáticas identificadas proveen una base para un modelo emergente, previsto para ser útil en la fase de diseño y planeación, y para la evaluación y monitoreo de los proyectos ICT4D.

9 Department of Computer and Systems Sciences, Stockholm University, Forum 100, 16440 Kista, Sweden. Email: mozelius@dsv.su.se

10 Department of Computer and Systems Sciences, Stockholm University, Forum 100, 16440 Kista, Sweden. Email: henrik.hansson@dsv.su.se

11 Department of Computer and Systems Sciences, Stockholm University, Forum 100, 16440 Kista, Sweden. Email: kahiigi@dsv.su.se

12 Department of Computer and Systems Sciences, Stockholm University, Forum 100, 16440 Kista, Sweden. Email: lovek@dsv.su.se 
Palabras clave: ІСт4D, proyectos de desarrollo, aspectos de éxito, evaluación de la calidad.

\begin{abstract}
Information and Communication Technology for Development (ICT4D) is an approach adopted to improve the socio-economic situation in poor countries. It includes e-learning, open and free software, e-democracy, digital media, e-government, capacity building and infrastructure development. Billions of dollars are invested in such programs financed by international aid organizations, state funded aid agencies and multinational private companies. Well known problems are poor infrastructure, crime and poor adaptations to the socio-cultural context. It is a complex multidisciplinary, multi stakeholder and multimedia field with an emerging theoretical framework.
\end{abstract}

This paper identifies and examines important quality aspects of ICT4D projects and provides empirical ICT4D examples illustrating each aspect. The overall research question in this study is: What makes some ICT4D projects more successful than others?

Based on the authors own experience of projects in Sri Lanka, Uganda and Vietnam as well as analysis of other projects, the following quality aspects are considered as crucial for successful implementation of ICT in development projects: 1) Authentic local needs, 2) Local ownership, 3) Realistic limitations, 4) Competence network, 5) Communication strategy, 6) Planning horizon, 7) Documentation/measurable results, 8) Resources and sustainability and 9) Fun/Motivation.

The relevance of these aspects are discussed and exemplified in the article.

The thematic areas identified provide a basis for an emerging model, intended to be useful in the design and planning phase as well as in monitoring and evaluation of ICT4D projects.

Key words: ICT4D, development projects, success aspects, quality assessment.

Recibido: 19 de marzo de 2009

Aceptado: 17 de abril de 2009

\title{
INTRODUCCIÓN
}

El mejoramiento de la efectividad de la beneficencia es una prioridad principal de la comunidad global. Tanto donantes como recipientes quieren obtener los mejores resultados posibles de las inversiones realizadas, para poder alcanzar los resultados específicos deseados. Los objetivos generales de los trabajos de beneficencia desde una perspectiva general, se encuentran delineados en lo que fue acordado en los Objetivos del Milenio) Millenium 
Goals. ${ }^{13}$ La Declaración del Milenio de las Naciones Unidas, ${ }^{14}$ es un documento corto y concentrado en ocho secciones; la primera, declara específicamente valores y principios internacionales, y las siguientes siete son áreas temáticas con objetivos clave específicos para alcanzar en el año 2015. Jefes de estado, ${ }^{15} 189$ en total, han acordado cumplir con los objetivos específicos relacionados con: 1) paz, seguridad, y desarme; 2) desarrollo y erradicación de la pobreza; 3) protección de nuestro ambiente común; 4) derechos humanos, democracia y buen gobierno; 5) protección de los vulnerables; 6) abarca las necesidades especiales de África; 7) fortalecimiento de las Naciones Unidas.

Un nuevo paradigma, cambiando la visión de cómo se deben llevar a cabo los proyectos de beneficencia, ha sido desarrollado en años recientes, particularmente manifestado en la Agenda de París (2005) la cual ha tenido gran aceptación; y en la Administración de Resultados de Desarrollo (Managing for Development Results -MfDR), enfoque dado por OECD Directiva de la Cooperativa por el Desarrollo (Development Cooperation Directorate -DCD-DAC) (2008). La agenda de París especifica doce indicadores de progreso para ser medidos nacionalmente y monitoreados internacionalmente. Estos indicadores se encuentran categorizados en cinco áreas temáticas: 1) Propiedad; 2) Alineación; 3) Armonización; 4) Administración de resultados, 5) Responsabilidad Mutua.

El enfoque MfDR significa: «...enfocarse en impactos concretos en todas las fases del proceso de desarrollo nacional. Una estructura concreta y un grupo de herramientas han sido desarrolladas para mejorar la participación gubernamental y para incrementar la responsabilidad en todos los niveles. El enfoque MfDR personifica principios generales aceptados de buen gobierno -fijando objetivos claros, la toma de decisiones basadas en evidencia, transparencia, y de adaptación y mejora continua.» (Managing for Development Results, 2008: 1).

Las evaluaciones son necesarias para las decisiones racionales en la continuación de proyectos presentes y para la toma de decisiones de proyectos futuros. Por ejemplo, la falta de fondos para el desarrollo general, la toma de decisiones en la formulación de políticas y la administración pública, son fuertemente dependientes de la evaluación continua del impacto de los conceptos de desarrollo, herramientas, actividades, y resultados. Más allá, un marco general es indispensable en la evaluación de factores de desarrollo, o la evaluación de la importancia de un proyecto en términos de su eficiencia, efectividad y sostenibilidad. El hecho de que las inversiones pueden llegar a tener un impacto en la relación entre costo y resultado debe ser evaluado de antemano. Algunos intentos han sido llevados a cabo para otorgar marcos particulares y generales para la evaluación y para sugerir métodos instrumentales para la asesoría de proyectos.

13 Millenium Goals, 07/09/2008.

14 United Nation, 2002.

15 End of Poverty, 07/09/2008. 
Sin embargo, un problema general al tomar cualquier decisión de experiencias previas, es que no mucho ha sido llevado a cabo de antemano para facilitar una evaluación sistemática de los varios proyectos. En este ensayo se usa un enfoque sistemático empírico en un campo que normalmente es discutido desde una perspectiva teorética, deductiva, o reguladora.

\section{OBJETIVOS}

El objetivo principal de este estudio es el de contribuir a un modelo útil para la aseguración de calidad de proyectos ict en países en vías de desarrollo. Un grupo de guías es generado con base en el análisis de casos seleccionados. La intención es la de identificar aspectos de calidad relevantes para todo el ciclo de vida del proyecto, desde la fase de preestudio hasta la evaluación final de un proyecto.

\section{Métodos y materiales}

Este ensayo se basa en el estudio de los siguientes casos: 1) El proyecto AsianLink/eBIT fundado por la Unión Europea en Sri Lanka, el cual se enfoca en un curriculum de desarrollo de e-learning; 2) Un estudio en la Universidad Makerere en Uganda sobre el uso y la experiencia de ICT; y 3) Una evaluación de SIDA - proyecto ICT para apoyar a los impedidos visualmente en Vietnam. Una mención general de los materiales y métodos utilizados se presenta en la siguiente tabla.

Tabla 1. Casos, datos y métodos

\begin{tabular}{|l|l|l|l|l|}
\hline Caso & País & Fuente de datos & Informantes & Métodos \\
\hline 1. AsianLink/eBit & $\begin{array}{l}\text { Sri } \\
\text { Lanka }\end{array}$ & $\begin{array}{l}\text { Estudios de campo } \\
\text { realizados por } \\
\text { Mozelius 2006- } \\
2008\end{array}$ & $\begin{array}{l}\text { Expertos en la } \\
\text { materia, diseñadores } \\
\text { institucionales, } \\
\text { desarrolladores de } \\
\text { contenido }\end{array}$ & $\begin{array}{l}\text { Observaciones } \\
\text { ytrevistas }\end{array}$ \\
\hline $\begin{array}{l}\text { 2. Universidad } \\
\text { Makerere }\end{array}$ & Uganda & $\begin{array}{l}\text { Estudios de campo } \\
\text { realizados por } \\
\text { Hansson \& Kahigi } \\
\text { 2008 }\end{array}$ & $\begin{array}{l}\text { Maestros y } \\
\text { Profesores }\end{array}$ & Entrevistas \\
\hline $\begin{array}{l}\text { 3. Entrenamiento ICT } \\
\text { para los } \\
\text { discapacitados } \\
\text { visuales }\end{array}$ & Vietnam & $\begin{array}{l}\text { Reporte de } \\
\text { Evaluación } \\
\text { realizado por } \\
\text { Mozelius, Nilsson } \\
\text { \& Nilsson, 2007. } \\
\text { Estudios de campo } \\
\text { por Mozelius } \\
\text { 2007. }\end{array}$ & $\begin{array}{l}\text { Maestros, } \\
\text { estudiantes, } \\
\text { personas encargadas } \\
\text { de la toma de } \\
\text { decisiones, } \\
\text { representantes de } \\
\text { organizaciones } \\
\text { locales. }\end{array}$ & $\begin{array}{l}\text { Observaciones, } \\
\text { entrevistas, } \\
\text { análisis de } \\
\text { documentos del } \\
\text { proyecto. }\end{array}$ \\
\hline
\end{tabular}

Procedimiento para la recopilación de datos: AsiaLink/eBIT, Sri Lanka.

Durante siete visitas a Sri Lanka entre 2006 y 2008, alrededor de quince conferencias, treinta talleres, y tres cuestionarios han sido la base para discusiones futuras con el Decano UCSC, expertos en la materia, diseñadores de instrucciones, desarrolladores de contenido, personal administrativo y estudiantes. En agosto 2006 y en mayo 2007, los participantes del proyecto 
sueco fueron invitados a una reunión con representantes para eBit facilitando lugares. En el programa eBit, la enseñanza como tal y gran parte de la felicitación, es llevada a cabo en lugares privados alrededor de la isla. Durante mayo 2007 y julio 2008, expertos suecos visitaron los lugares de facilitación y telecentros, y llevaron a cabo entrevistas informales con el personal y los estudiantes.

A distancia, vía internet, los participantes suecos han formado parte de los foros de discusión virtuales en Sri Lanka donde temas relacionados con los cursos eBIT han sido discutidos. Las discusiones han sido, principalmente, llevadas a cabo en el ambiente virtual para el aprendizaje Moodle. ${ }^{16}$ En la lucha hacia contacto regular, las reuniones del proyecto han sido llevadas a cabo a larga distancia, usando un sistema de conferencia virtual Polycom. ${ }^{17}$ En varias ocasiones, personas suecas han asistido a reuniones de personal y seminarios internos de desarrollo. Un ejemplo es la conferencia para el personal eBIT para el desarrollo de cursos en el cuarto semestre eBIT, en Negombo, marzo 2008. Datos fueron recopilados de los informantes mencionados anteriormente para proveer una visión válida y balanceada de la implementación, el impacto y la relevancia de los proyectos. Problemas y cuestiones críticos fueron analizados y discutidos de forma particular.

\section{Procedimiento de la recolección de datos: Universidad Makerere, Uganda}

Entrevistas informales fueron llevadas a cabo en la Universidad Makerere con cuatro conferencistas y once estudiantes. Los conferencistas entrevistados estaban relacionados con el Instituto de Salud Pública (Institute of Public Health), el Departamento de Odontología (Department of Dentistry), la facultad de Educación y la Facultad de Computación y Tecnología. Los estudiantes estaban relacionados con la Facultad de Economía, Facultad de Computación y Tecnología y el Instituto de Psicología.

El objetivo de las entrevistas constituía dos aspectos: el primero, desarrollar un entendimiento de lo que es considerado base en relación con la utilidad que se percibe del uso de la enseñanza y de actividades educacionales basadas en e-learning; el segundo, proveer información que será utilizada para notificar el diseño del plan de estudio exploratorio empírico. Sin embargo, vale la pena notar que los resultados obtenidos, hasta ahora, no son plenamente representativos y entrevistas continuas serán llevadas a cabo en un estudio de seguimiento. Los resultados obtenidos después de entrevistas sustantivas guiarán el desarrollo de un modelo para la utilización efectiva de e-learning para apoyar actividades de aprendizaje y enseñanza en el contexto de educación superior.

La construcción principal de la discusión llevada a cabo con conferencistas y estudiantes se basó en la utilidad que percibían en el uso de e-learning y cómo era dicho uso en sus actividades de enseñanzas y aprendizaje. Sin embargo, de las discusiones que tuvieron lugar mientras la infraestructura para apoyar e-learning estaba ya en uso, hubo poco agarre

\footnotetext{
16 Moodle, 24/09/2008.
}

17 Polycom, 24/09/2008. 
relacionado con e-learning. Las discusiones se centraban en los problemas que se debían superar al adoptar el uso de e-learning en sus actividades de enseñanza y aprendizaje.

\section{Procedimiento para la recolección de datos: entrenamiento ICT para los discapacitados visuales. Vietnam}

Durante una visita de catorce días a Vietnam en septiembre de 2007, entrevistas formales fueron realizadas con profesores, estudiantes, y personal en las ramas vBA en Hanoi, Hai Phong y en la ciudad de Ho Chi Minh. Entrevistas profundas, y discusiones más informales fueron llevadas en la escuela NCD en Hanoi. Cuestionarios fueron enviados a personas seleccionadas de antemano vía correo electrónico desde Suecia. En Suecia, entrevistas profundas fueron realizadas con participantes de Iris Hadar y de la Organización Sueca para los discapacitados visuales (Swedish Organization for the Visually Impaired-SRF).

La mayor parte de la documentación del proyecto fue analizada y seguida por correos electrónicos con preguntas a los participantes de los proyectos. Los evaluadores asistieron a las reuniones y discutieron el proyecto con las personas involucradas con Sida, SRF, e Iris Hadar en Suecia; y con el personal de vBA, la escuela NCD, y la embajada de Suecia en Vietnam.

Los diversos casos seleccionados proveen una base para la generalización inductiva. Usamos el marco analítico de teoría basada originalmente y desarrollada por Barney Glaser y Anselm Strauss,$^{18}$ con su enfoque guiando los principios del análisis de datos. ${ }^{19}$ El punto de partida es el de comenzar con datos, no el de comprobar una hipótesis. El proceso de análisis consiste en codificar información clave, abstrayéndola de conceptos centrales y categorías y, finalmente, crear el modelo con las áreas temáticas más importantes. No es un proceso linear desde los datos hasta los conceptos generales, sí un proceso interactivo, donde los datos generan ideas para conceptos centrales y los conceptos tentativos son comprobados con el material empírico.

\section{RESULTADOS Y DISCUSIONES}

\section{AsiaLink/ eBIT, un modelo para aprendizaje basado en la red}

Este es un proyecto en el marco de la Unión Europea que ahora se encuentra en su fase final. Una expectativa real es que el factor más importante de los objetivos específicos, el de aumentar el margen de aprobados en la Universidad de Colombo, Facultad de Computación (UCSC) ${ }^{20}$ en programación eBIT, sea alcanzado. Una de las tantas razones para el buen trabajo del lado de Sri Lanka es la relevancia del primer objetivo global: «Satisfacer algunas de las demandas de trabajo creadas en Sri Lanka por la expansión de los sectores económicos relacionados con ICT.»»

18 Glasser y Strauss (2206/1967).

19 Véase también Charmaz (2006); Dey (1999); Glaser (1998).

20 USCS, 03/07/2008.

21 AsiaLink/eBIT, 01/07/2008. 
A menudo, es mucho más fácil tener éxito cuando la economía está creciendo y cuando existen objetivos claros y reales, no obstante debe ser en cooperación con expertos locales hábiles y comprometidos con el equipo de proyecto. Una combinación, que en ocasiones se muestra también contradictoria, es la de las universidades en las cuales los salarios son significativamente más bajos que en el sector ICT. No es siempre el caso, que la gente hace lo que hizo el doctor K. P. Hewagamage, cuando regresó de Japón a UCsC para guiar la construcción de un programa de aprendizaje e-learning nacional. ${ }^{22}$ Es de gran valor tener a alguien que se preocupa por las actividades los 365 días del año.

Otra tarea importante es la de cómo organizar una red internacional de excelencia y evadir muchas generalidades en la actividad. No hay una solución fácil cuando el campo ICT atrae también a muchos de los profesionales más habilidosos en los países desarrollados. Es necesario dividir las responsabilidades y no tratar de resolver muchos problemas al mismo tiempo. En el proyecto de AsiaLink/eBIT, la parte pedagógica es organizada por la Universidad de Estocolmo, Departamento de Computación y Sistemas Científicos (DVS) ${ }^{23}$ y el mejoramiento de la evaluación en línea es llevado a cabo por la Universidad Delft de Tecnología (DUT). ${ }^{24}$ Una experiencia del proyecto eBIT es que mientras más crece la red de recursos humanos, mayor es la necesidad de una documentación común.

El proyecto eBIT es la continuación de una serie de proyectos suecos de beneficencia donde experiencias tempranas y contactos previos facilitan la planeación a largo plazo y añaden agilidad a la colaboración. Después de la finalización del proyecto eBIT el intercambio Suecia-Sri Lanka será continuado en $\operatorname{Sida}^{25}$ proyecto fundado durante la construcción de un centro e-learning en Sri Lanka.

Impresiones generales. Excepto por el hecho que Sri Lanka se encuentra en una situación cercana a la guerra civil, ambos proyectos, el de beneficencia y el de desarrollo ICT se encuentran en buenas condiciones en el país. La serie de proyectos ICT de beneficencia, no solo de Europa, han ciertamente tenido un efecto. En el proyecto AsianLink/eBIT el objetivo de incrementar en número de aprobados de un 2 a un $5 \%$ en los cursos e-learning va a ser más que alcanzado y la producción de contenidos en estos cursos ha alcanzado un nivel donde UCSC ha comenzado a exportar e-learning. Durante los últimos años, UCSC ha comenzado a arreglar conferencias ICT у cursos de entrenamiento para participantes de otros países en desarrollo. Al mismo tiempo que Sri Lanka en los años venideros abandonará el término de país en vías de desarrollo, el centro e-learning y el programa eBIT tendrán grandes oportunidades de continuar su existencia, ser sostenibles y continuar su expansión.

22 Hewagamage, 03/07/2008.

23 DSV, 05/07/2008.

24 Delft DUT, 05/07/2008.

25 La Agencia Internacional de Cooperación para el Desarrollo, the Swedish International Development Cooperation Agency. 


\section{Percepción de los estudiantes y de los conferencistas acerca del aprendizaje $\boldsymbol{e}$-learning en la Universidad Makerere, Uganda}

La Universidad de Makerere implementó un proyecto e-learning fundado por la Coorporation Carnegie, Nueva York. El objetivo del proyecto es el de asegurar graduandos de calidad, para el sector público y el privado, con habilidades claves para asegurar que Uganda se junte con las sociedades del conocimiento. La universidad adoptó un enfoque mixto con un gran porcentaje de compromiso cara a cara. Hasta el día de hoy, el progreso ha sido en términos de proveer la infraestructura, con retos en el sentido humano relacionados con la falta de personal, perspectiva de entrenamiento, entre otros. La universidad está actualmente utilizando Blackboard y KEWL (fuente abierta) como sus sistemas de administración del aprendizaje.

Perspectiva de los conferencistas: los conferencistas trajeron a colación las siguientes preocupaciones en relación a la adopción de e-learning en sus iniciativas de enseñanza:

- Un gran número de estudiantes presenta un reto relacionado con el apoyo y manejo en red de los estudiantes. El conferencista: la proporción de estudiantes en algunos cases es 1:800, sin asistentes que los ayuden a administrar los cursos, incluso en el modo tradicional presencial.

- El acceso a computadoras es otro tema que afecta la adopción del sistema e-learning en la Universidad Makerere. Mientras que la Universidad continúa en actitud de proveer acceso a computadoras, aún no hay suficientes para los estudiantes. En este caso, los conferencistas vacilan en el desarrollo de cursos en línea ya que piensan que esto sería un esfuerzo perdido, pues los estudiantes no podrían acceder a los cursos.

- Falta de habilidades computacionales es otro tema que fue traído a colación. La mayoría de los conferencistas y los estudiantes no tienen las habilidades que son imperativas para utilizar con efectividad las tecnologías en sus actividades de enseñanza y aprendizaje. Esto ha afectado de gran manera la forma como se ve el e-learning ya que los estudiantes no pueden contar con el apoyo de los conferencistas en un ambiente en línea. Mientras que los estudiantes, en algunos casos tienen apoyo de sus semejantes, los conferencistas, especialmente las generaciones más viejas, no tienen el entusiasmo de adquirir estas habilidades. Los conferencistas también notaron una falta de habilidades para integrar los aspectos pedagógicos con las tecnologías para así apoyar efectivamente el aprendizaje del estudiante.

- La falta de tiempo para desarrollar y administrar cursos en línea fue otro de los temas mencionados. Los conferencistas sintieron que no tenían el tiempo adecuado para administrar cursos en línea.

- Falta de apoyo administrativo para encabezar el desarrollo de e-learning desde la unidad hasta el nivel administrativo más alto. Los conferencistas señalaron que a nivel individual los esfuerzos para desarrollar cursos en línea no son reconocidos y son vistos como personales en lugar de iniciativas unitarias. Ahora, al nivel más alto administrativo, la política no ha sido puesta en funcionamiento, lo que lleva a la renuencia en la adopción del aprendizaje e-learning. 
Perspectiva delos estudiantes: Los estudiantes trajeron a colación las siguientes preocupaciones relacionadas con la adopción de e-learning en sus actividades de aprendizaje:

- Falta apoyo del tutor en los ambientes presenciales y en línea. Esto fue atribuido al gran número de estudiantes que abruman a los conferencistas. Los estudiantes mencionaron que los conferencistas proveen apoyo limitado al final de las clases, mediante una sección de preguntas y respuestas. Aclaraciones fuera de clase se pueden obtener a través de la discusión entre estudiantes, con contacto limitado, y en muchos casos inexistente, con el profesor conferencista. Esto fue afirmado por uno de los conferencistas entrevistados quien estableció que «....Hay muchos estudiantes en mi clase. Yo no puedo responder a todas sus preguntas durante las sesiones de discusión asignadas. También les doy una hora de consulta semanal una vez a la semana; sin embargo, no los puedo ver a todos durante este tiempo...».

- Los estudiantes también mencionaron la falta de habilidades computacionales de los conferencistas para guiar la adopción de e-learning en sus actividades de enseñanza. Respecto a esto, uno de los estudiantes dice que «...los conferencistas deben estar informados acerca de los desarrollos más recientes en tecnologías que apoyarán el aprendizaje estudiantil...».

- Los estudiantes también hacen mención de la falta de salas e instalaciones de computadoras para acceder a los recursos en línea.

Sin embargo, mientras que muchos de los retos relacionados con la adopción de e-learning fueron mencionados, los estudiantes también dieron su opinión acerca del valor adicional que se obtendría del uso de tecnologías para apoyar sus actividades de enseñanza y aprendizaje, dentro del cual incluían:

- Tener acceso al material de estudio

- Compartir información y discusiones

- Lograr habilidades que lleven a diversas oportunidades

- Poder monitorear el desarrollo personal de los estudiantes

Impresiones generales. Es evidente que los profesores perciben generalmente ICT como una carga adicional que consume mucho tiempo. Los hábitos de los conferencistas también afectan el estilo de enseñanza no cambiante, ya que pueden dar repetidamente la misma clase o charla, sin mucha interacción semana tras semana, y no deben considerar un modo centrado más en los estudiantes. También fue obvio que los estudiantes tenían una gran necesidad de interacción y comunicación para poder resolver sus tareas y entender las clases y charlas. Un «grupo de apoyo de emergencia estudiantil» ha sido formado espontáneamente entre alrededor de sesenta estudiantes, quienes a través de teléfonos celulares, computadoras y reuniones se comunican para resolver sus asignaciones universitarias e interpretar las intenciones del conferencista, el material, y las clases. Las plataformas y herramientas ICT dadas al personal no eran utilizadas, pero tenían una gran demanda por parte de los estudiantes, quienes, sin embargo, no podían utilizarlas. 


\section{Sida/vBA, entrenamiento ICT para los discapacitados visuales en Vietnam}

Muy a menudo, los proyectos son iniciados por una persona entusiasta con un grande deseo de hacer que algo se vuelva realidad. Este fue el caso del proyecto Sida para el apoyo ICT para los discapacitados visuales en Vietnam, cuando la artista sueca Elisabeth Persson quería apoyo digital para sus proyectos de arte en la escuela Nguyen Dinh Chieu para ciegos en Hanoi. Más tarde, sus ideas fueron elaboradas y extendidas por Sida, la Asociación Sueca para los Discapacitados Visuales en Halland y la Asociación de Ciegos de Vietnam (Vietnam Blind Association), pero debe existir alguien que tome el primer paso.

La idea de agregar arte y música en un proyecto para entrenamiento computacional básico funcionó muy bien en la escuela NCD. En las otras escuelas participantes en Hanoi, Hai Phong y la ciudad de Ho Chi Minh el currículo de entrenamiento era un poco diferente pero lo que tienen en común es que las actividades del proyecto tuvieron un efecto positivo en las vidas diarias de las personas que participaron en el entrenamiento ICT. Este entrenamiento fue dado por la compañía sueca Iris Hadar con una pedagogía especial compuesta para el entrenamiento computacional para ciegos o discapacitados visuales. ${ }^{26}$ Incluso, aun si los muy ambiciosos objetivos del proyecto nunca fueron alcanzados, el impacto en un grupo que había sido previamente ignorado fue muy positivo, y el programa ICT de diez semanas ha sido reaplicado, prolongado y aumentado por profesores locales en las escuelas involucradas. ${ }^{27}$

De ser un proyecto originalmente sueco, el entrenamiento computacional y la propiedad del proyecto pasaron al lado vietnamés. Este proyecto podría ser correctamente criticado por un preestudio pobre y un esquema vago, pero algunas veces la falta de planeación puede ser compensada con compromiso y participación local. Pero lo que hubiese podido mejorar por una base bien definida, es la relación costo-eficiencia del proyecto.

Impresiones generales. Más que la identificación de una necesidad local, el proyecto fue iniciado por lo que es la suposición de una necesidad general global. A pesar de la eficacia de bajo costo, el impacto es obvio. En entrevistas profundas con los participantes del curso, $100 \%$ de los entrevistados respondieron que el curso había mejorado su estilo de vida, dándoles nuevos canales de información y nuevas herramientas de comunicación digital.

El hecho de que el objetivo principal de los proyectos estaba lejos de ser alcanzado debe ser visto más como un resultado de ambiciones poco realistas que de la mala administración del proyecto. El crear oportunidades de trabajo para los discapacitados visuales es una tarea ardua hasta en los países desarrollados. Todos los componentes de entrenamiento organizados por Iris Hadar estaban bien planeados y llevados a cabo de una manera amistosa y profesional. Todos los maestros entrevistados en Vietnam mencionaron que Iris Hadar realizó talleres de entrenamiento que los inspiraron para utilizar nuevas técnicas pedagógicas.

26 Iris Hadar, 06/07/2008.

27 Sida-Vietnam, 06/07/2008. 
Incluso si el resultado fue exitoso, la crítica del muy limitado preestudio se mantiene y el monitoreo y documentación fueron insuficientes.

\section{CONCLUSIONES}

Este ensayo se basa en el análisis de tres casos ICT4D. Por medio de generalizaciones empíricas de estos casos, se obtienen nueve importantes aspectos temáticos para considerar en la planeación e implementación de proyectos ICT4D (véase la siguiente figura). Los aspectos temáticos concluyentes son explicados en más detalle y relacionados con los casos a continuación.

Figura 1. Factores de importancia para proyectos ICT4D exitosos.

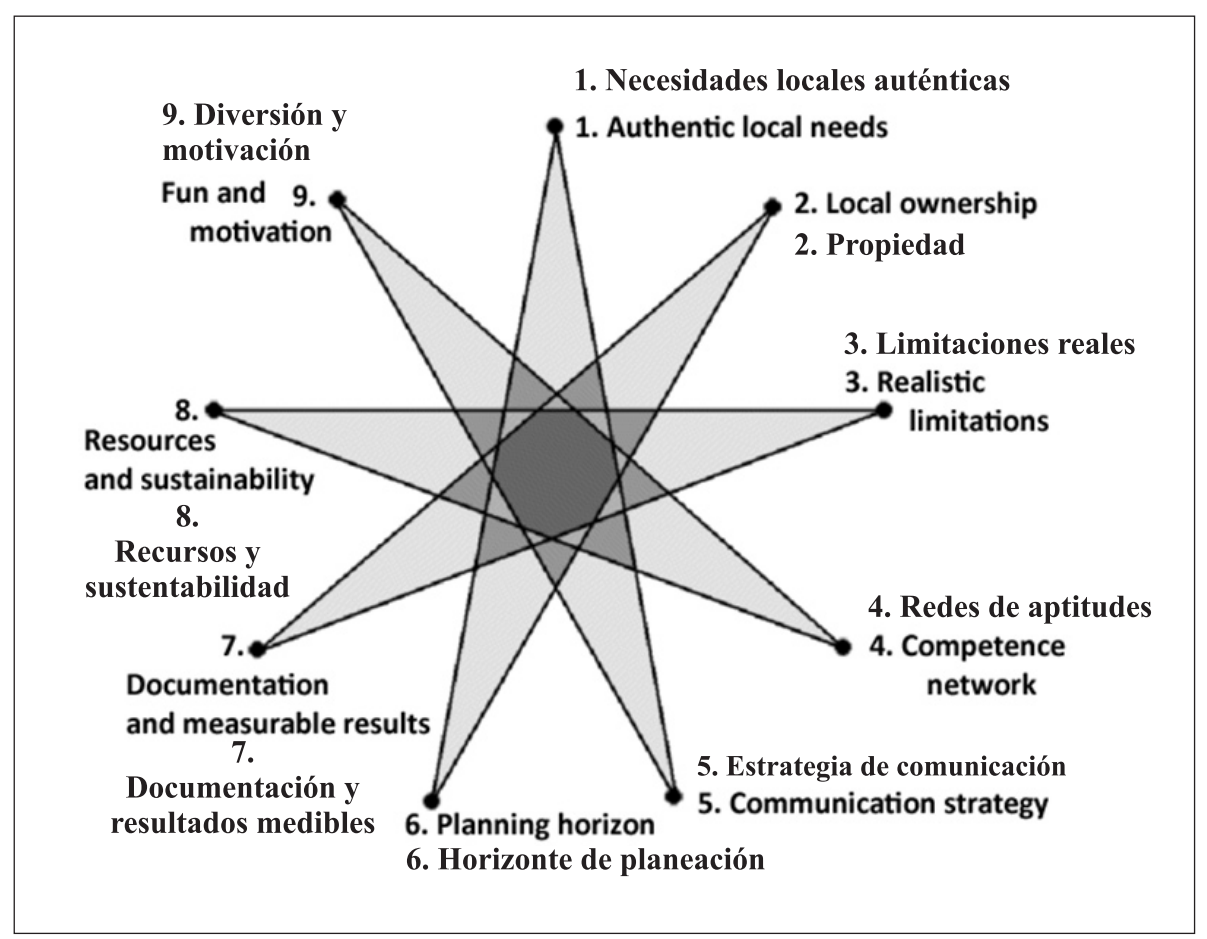

\section{Necesidades locales auténticas}

Si usted, como en Sri Lanka, tiene una indudable necesidad de una fuerza de trabajo en un campo en particular, un proyecto para una apropiada educación que satisfaga esta demanda debería ser adecuado. Pero, por supuesto, para satisfacer la necesidad principal, todas las necesidades relacionadas con la educación deben ser tratadas. En el caso de Uganda, las necesidades fueron claramente expresadas por los estudiantes, sin embargo, no todas fueron tomadas en cuenta por los profesores y, por lo tanto, la implementación no fue exitosa. 


\section{Propiedad local}

En Uganda, las herramientas ITC no eran apropiadas para las necesidades de los profesores, y estos no estaban entrenados en su uso. Las plataformas usadas fueron dadas por donantes internacionales y no estaban integradas realmente en el uso diario. La propiedad local era un factor importante para los Telecentros exitosos en Uganda, como se puedo concluir en un estudio anterior. ${ }^{28}$

\section{Limitaciones reales}

Satisfacer algunas de las demandas de la fuerza de trabajo en el sector ICT en Sri Lanka mediante la expansión del programa universitario existente de tres años parece tener una posibilidad razonable de éxito. El objetivo principal del apoyo para los discapacitados visuales en Vietnam era mucho más difícil de alcanzar. En Vietnam, el tiempo real de entrenamiento del grupo original, no era de más de diez semanas. Inclusive si la pedagogía y el plan de estudios trabajaban bien, no era tiempo suficiente para construir una base para un trabajo, sin importar la velocidad en la cual el campo ITC doméstico está creciendo.

\section{Redes de aptitudes}

Para hacer que un proyecto funcione, este debe soportarse en un equipo con todas las habilidades y conocimientos necesarios, y alternativamente un plan posible para el desarrollo de las habilidades faltantes dentro del proyecto. En Uganda existía la necesidad de enseñar a los profesores acerca del uso pedagógico de ICт. También el personal de apoyo necesitaba ser ampliado para poder implementar los ICT y apoyar mejor a los docentes.

\section{Estrategia de comunicación}

Un plan explícito y aceptado para el monitoreo de la comunicación es necesario: cómo, cuándo, y acerca de qué. Este aspecto es particularmente importante ya que las parejas en los proyectos ICT4D trabajan típicamente con interacciones a larga distancia y también enfrentan diferencias culturales e idiomas. Para evadir un monitoreo pobre, como en el caso vietnamés, asegurar las herramientas de comunicación web2.0 disponibles hoy en día podría mejorar la calidad sustancialmente. Los estudiantes de Uganda usaban sus propios teléfonos móviles e instalaciones de telecentro, pero, apoyar su comunicación creando plataformas y foros disponibles ampliaría sus capacidades de aprendizaje. Los profesores de Uganda necesitaban un modelo para comunicarse con un número de estudiantes más amplio de manera efectiva.

\section{Horizonte de planeación}

A menudo, se carece de planeación a mediano y largo plazo. Sin claros y explícitos planes de visita, actividades y objetivos, un proyecto no puede alcanzar su potencial. Esto, sin embargo, no significa que no deban ser hechas adaptaciones y modificaciones. La necesidad de un horizonte de planeación razonable, un aspecto de calidad general concluido de una amplia experiencia de proyectos, no sólo en ICT4D en proyectos de la Unión Europea. Las responsabilidades caen sobre todas las partes involucradas, pero en particular del coordinador. La planeación y la comunicación con una buena perspectiva del tiempo, siempre llevan a la inclusión de los participantes en la preparación y control de su situación de trabajo. En el

28 Hansson, Kivunike, Mozelius (2008). 
proyecto de Sri Lanka algunas actividades no han sido programadas con un amplio marco de tiempo, lo cual tuvo un impacto en el resultado.

\section{Documentación/resultados medibles}

El valor de documentación cuidadosa y accesible puede ser ilustrado con el caso vietnamés. Si se pretende que los paquetes de trabajos exitosos sean transferidos y rehusados, esto sería mucho más fácil si existiese documentación estructurada disponible y pública. Los proyectos ICT4D están, en la mayoría de los casos, enfocados en aspectos prácticos y en formas para hacerlos funcionar. Pero la documentación pobre y negligente de la implementación y resultados de proyectos no corresponde con lo acordado en la Agenda de París relacionado con transparencia y responsabilidad. Herramientas sencillas, tales como blogs ICT, wikis, y páginas de inicio, pueden ser utilizadas para propósitos de documentación.

\section{Recursos y sustentabilidad}

Mientras más se pueda transferir, en términos de la propiedad, del lado del donante al país recipiente, es mejor para la sostenibilidad. En el caso vietnamés, esto fue logrado mediante iniciativas personales no planificadas pero, es preferible que esto suceda y sea parte de una estrategia común.

\section{Diversión / Motivación}

Sin alegría, entusiasmo, y compromiso, un proyecto tiene menos probabilidades de ser eficiente y exitoso. La creación de redes sociales entre los participantes debe ser recomendada. En Uganda, los estudiantes expresan un fuerte compromiso, y alegría al colaborar. Estructuras con menos jerarquía dan poder a las personas y liberan energía. Sin embargo, las diferentes responsabilidades deben ser asignadas a cada individuo.

\section{Estudios futuros y desarrollo}

Obviamente, este estudio tiene una fuerte perspectiva empírica, resaltando algunos factores importantes para análisis futuros. Una línea importante de investigación futura sería el poner a prueba proyectos planeados, en funcionamiento, o completados, de una selección más amplia de casos. Para desarrollar un marco entero y comprobado, la inclusión de enfoques cualitativos y cuantitativos más elaborados es deseable así como también, y en particular, un modelo y un procedimiento analítico de multicriterios, que sea capaz de manejar argumentos cualitativos y cuantitativos. Este marco estaría basado en nuestra investigación más temprana acerca de la maquinaria evaluativa para este tipo de problemas y facilitaría considerablemente una provocación de partes interesadas y de estructuras de valores.

\section{REFERENCIAS BIBLIOGRÁFICAS}

CHARMAZ, K. Constructing grounded theory: a practical guide through qualitative analysis. London: SAGE. 2006.

DEY, I. Grounding grounded theory: guidelines for qualitative inquiry. San Diego: Academic Press. 1999. 
GLASER, B, G. Doing grounded theory: issues and discussions. Mill Valley, Calif:. Sociology Press. 1998.

GLASER, B, G, STRAUSS, A, L. The discovery of grounded theory: strategies for qualitative research. New Brunswick, N. J.: Aldine Transaction (a division of Transaction Publishers). 2006, c1967.

HANSSON, H, KIVUNIKE F, N, MOZELIUS, P. An Analysis of Best and Worst Practices in Aid Projects. Presentation at the IPID ICT4D PG symposium 2008. September 7-9, Joensuu, Finland. 2008.

OECD Development Co-operation Directorate (DCD-DAC) (2008). Managing for development results. Information sheet. (2008).

UNITED NATIONS. Resolution adopted by the General Assembly.[without reference to a Main Committee (A/55/L.2)]. 55/2. United Nations Millennium Declaration. 8th plenary meeting 8 September 2000 .

\section{FUENTES ELECTRÓNICAS}

AsiaLink/eBIT, 01/07/2008. <http://www.dellka.ec.europa.eu/en/eu_and_srilanka/projects/ asia_ebit.htm>.

Delft DUT, 05/072008. <http://www.citg.tudelft.nl>

DSV, 05/07/2008. <http://dsv.su.se/en>

Hewagamage, 03/07/2008. <http://www.ucsc.cmb.ac.lk/People/kph>

Iris Hadar, 06/07/2008. <http://www.dinf.ne.jp/doc/english/prompt/ws051115_lm_s. html\#startreading>

Moodle, 24/09/2008. <http://moodle.org>

Overview aboutthemillennium developmentgoals, 07/09/2008. $<$ http://www.endpoverty2015. org/goals>

Polycom, 24/09/2008. <http://www.polycom.com/apac/en/home/index.html>

Sida-Vietnam, 06/07/2008. $<$ http://www.scandasia.com/viewNews.php?news_id=4437\&coun_code=se >

The Paris Agenda. Indicators of Progress. To be measured nationally and monitored internationally, 07/09/2008. <http://www.oecd.org/dataoecd/57/60/36080258.pdf>

USCS, 03/07/2008. <http://www.ucsc.cmb.ac.lk> 